\title{
Uptake and Accumulation of Nephrotoxic and Carcinogenic Aristolochic Acids in Food Crops Grown in Aristolochia clematitis-Contaminated Soil and Water
}

Weiwei $\mathrm{Li}$, Qin $\mathrm{Hu}$, and Wan Chan*

Department of Chemistry, The Hong Kong University of Science and Technology,

Clear Water Bay, Kowloon, Hong Kong SAR, China

* Corresponding author:

Email: chanwan@ust.hk, Tel: (852) 2358-7370,Fax: (852) 2358-1594 


\section{FIGURE LEGEND}

Figure S1: UPLC-MS analyses of aristolactam I $(\mathrm{m} / \mathrm{z}=294)$ from the nitroreduction of (A) AAI standard and (B) AAI in red oak lettuce samples, together with chromatograms from the UPLC-MS analyses of aristolactam II $(m / z=264)$ from the nitroreduction of (C) AAII standard and (D) AAII in the red oak lettuce sample. The insets show the product ion spectra of the $[\mathrm{M}+\mathrm{H}]^{+}$ion of aristolactams.

Figure S2: HPLC-FLD analyses of AAI and AAII in lettuce (butterhead) grown in AA contaminated soil. The nitroreduction product of AAI and AAII were eluted for 10.6 and $10.2 \mathrm{~min}$, respectively.

Figure S3: Concentrations of AAI and AAII in butterhead lettuce grown in AA-contaminated soil. Lettuce plants were irrigated daily with herbal extracts of Aristolochia clematitis from day 1 to day 21 and samples were collected weekly for HPLC-FLD analyses.

Figure S4: HPLC-FLD analyses of aristolactams and AAs in herbal extracts of Aristolochia clematitis. Aristolactam I and aristolactam II were eluted at $10.6 \mathrm{~min}$ and $10.2 \mathrm{~min}$, respectively. The concentrations of AAs were determined using the method described in (Figure 2).

Figure S5: Concentrations of aristolactam I and aristolactam II in butterhead lettuce grown in AA-contaminated soil. Lettuce plants were irrigated daily with herbal extracts of Aristolochia clematitis from day 1 to day 21 and samples were collected weekly for HPLC-FLD analyses.

Figure S6: Concentrations of AAs in AA-spiked culture medium with no lettuce growing on it. 
Figure S1
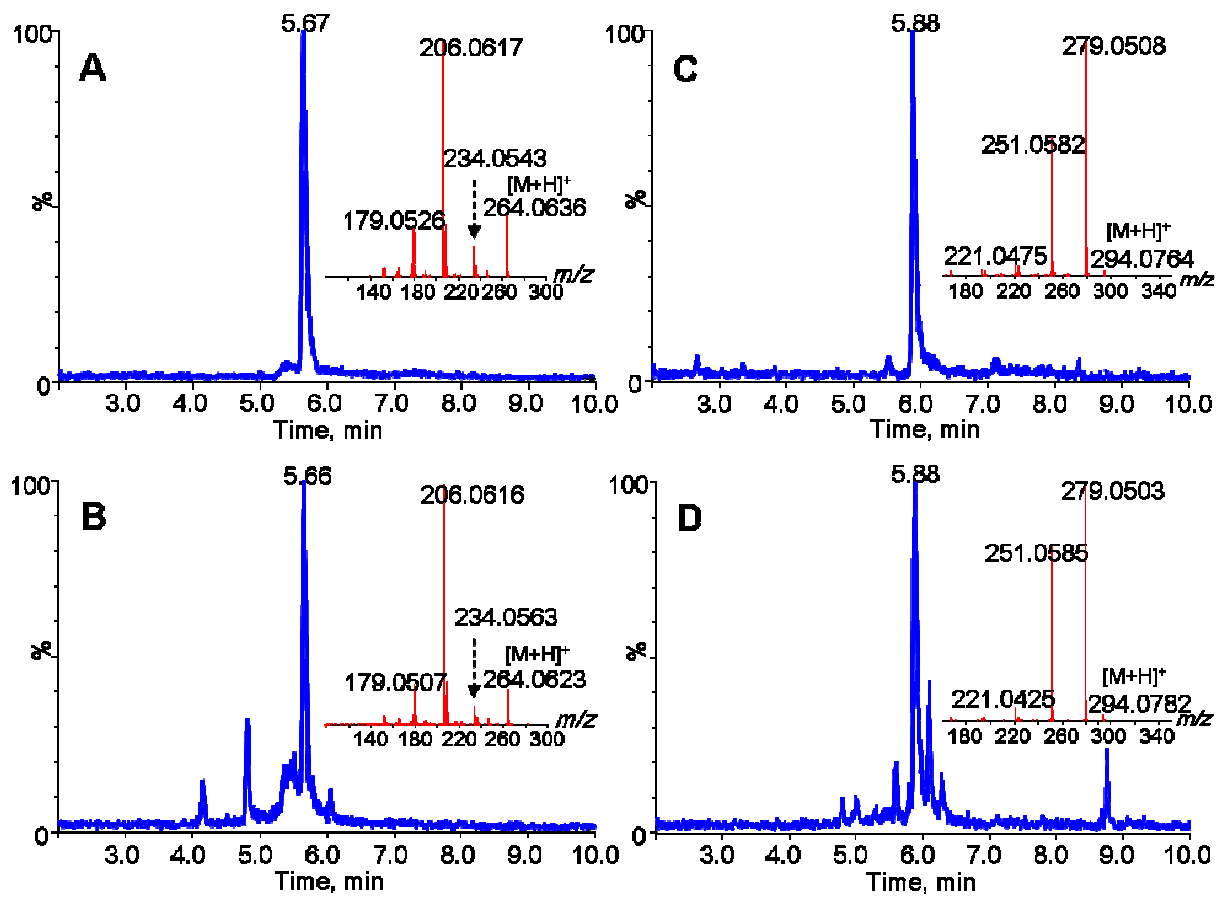
Figure S2

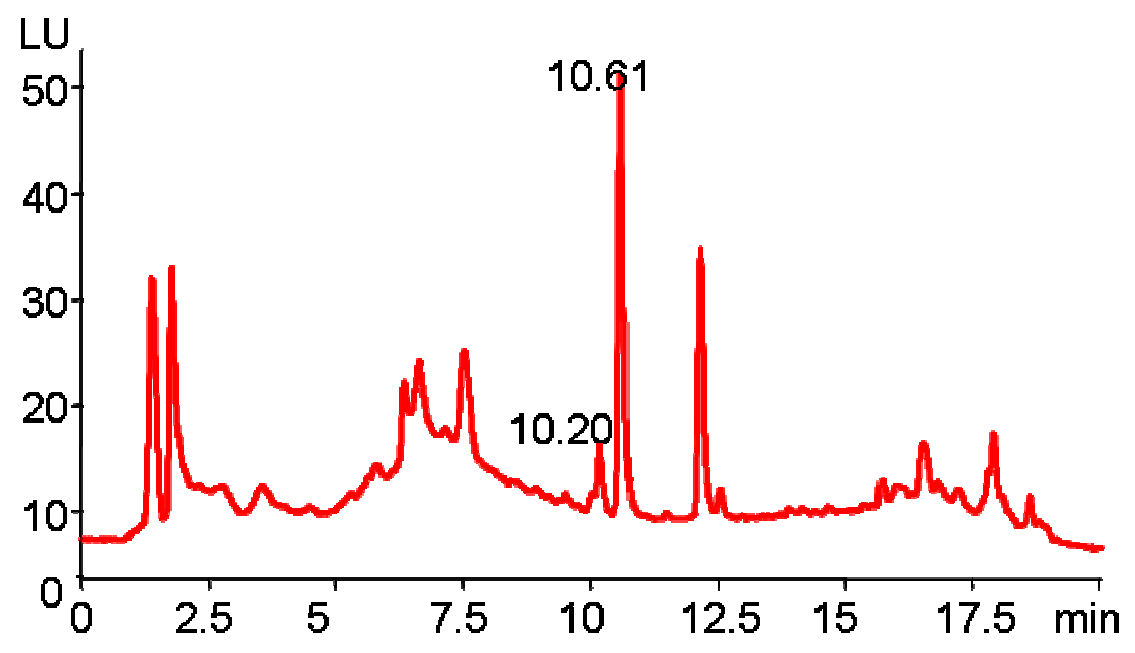


Figure S3

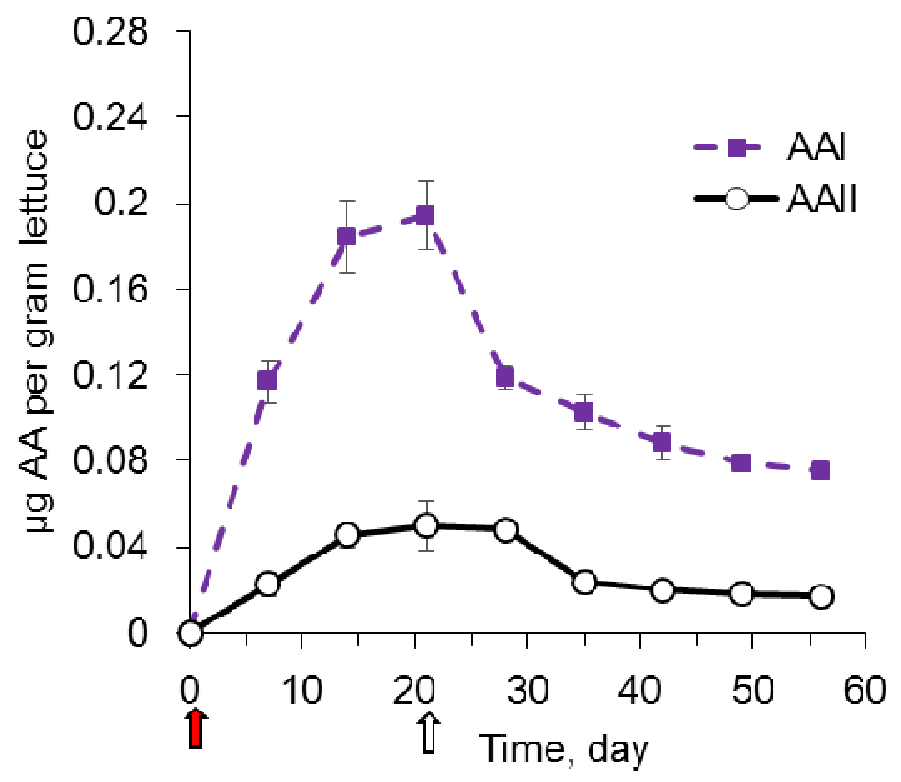


Figure S4

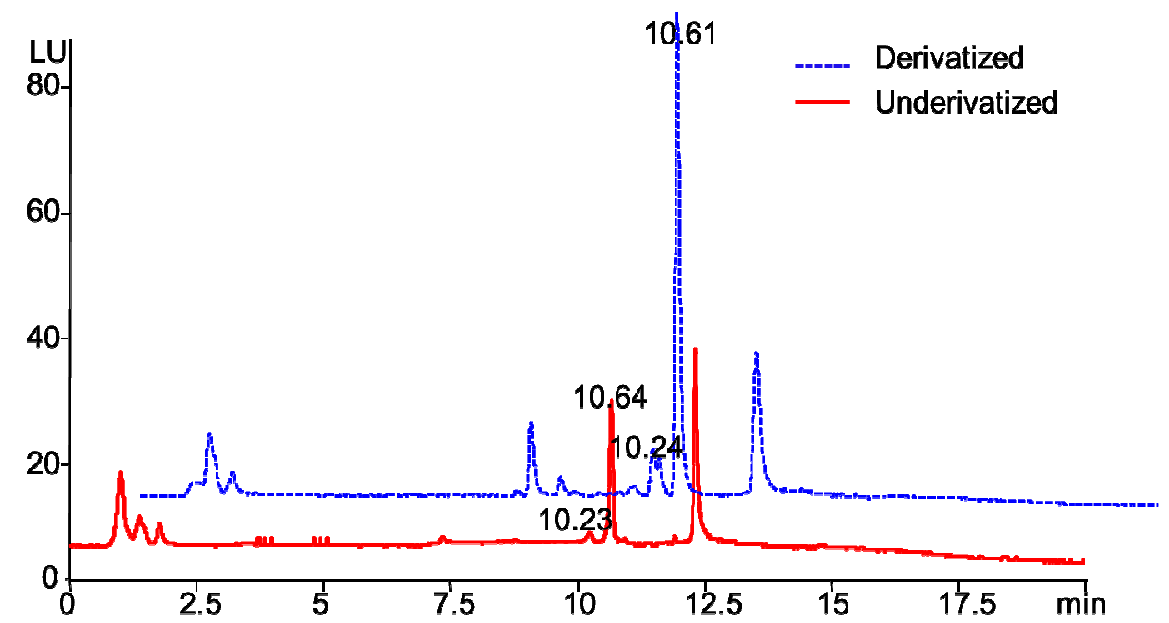


Figure S5

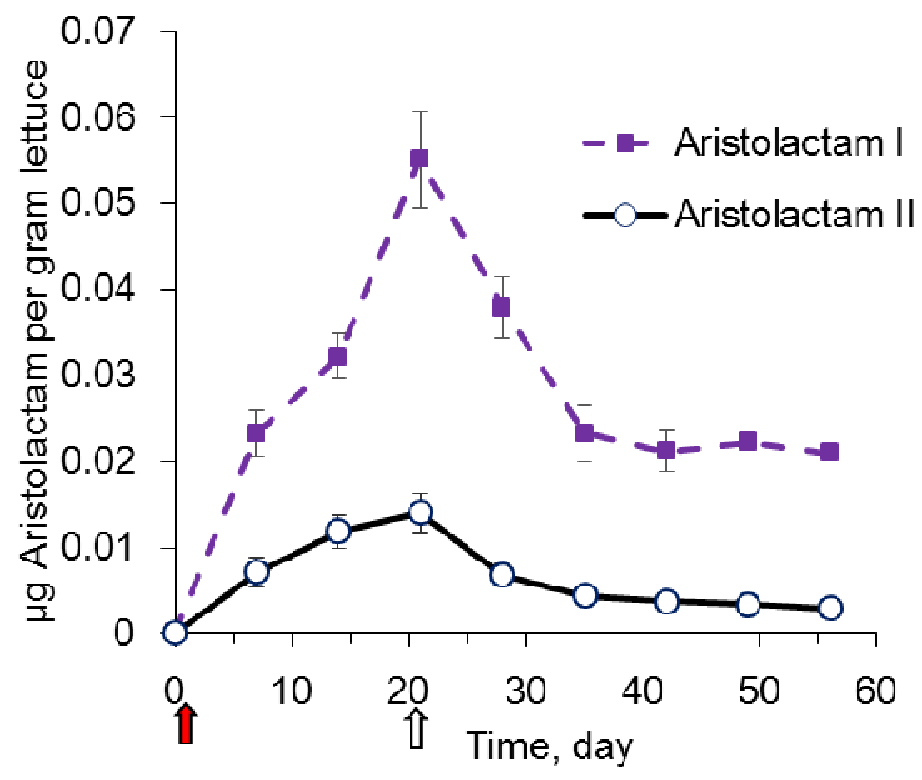


Figure S6
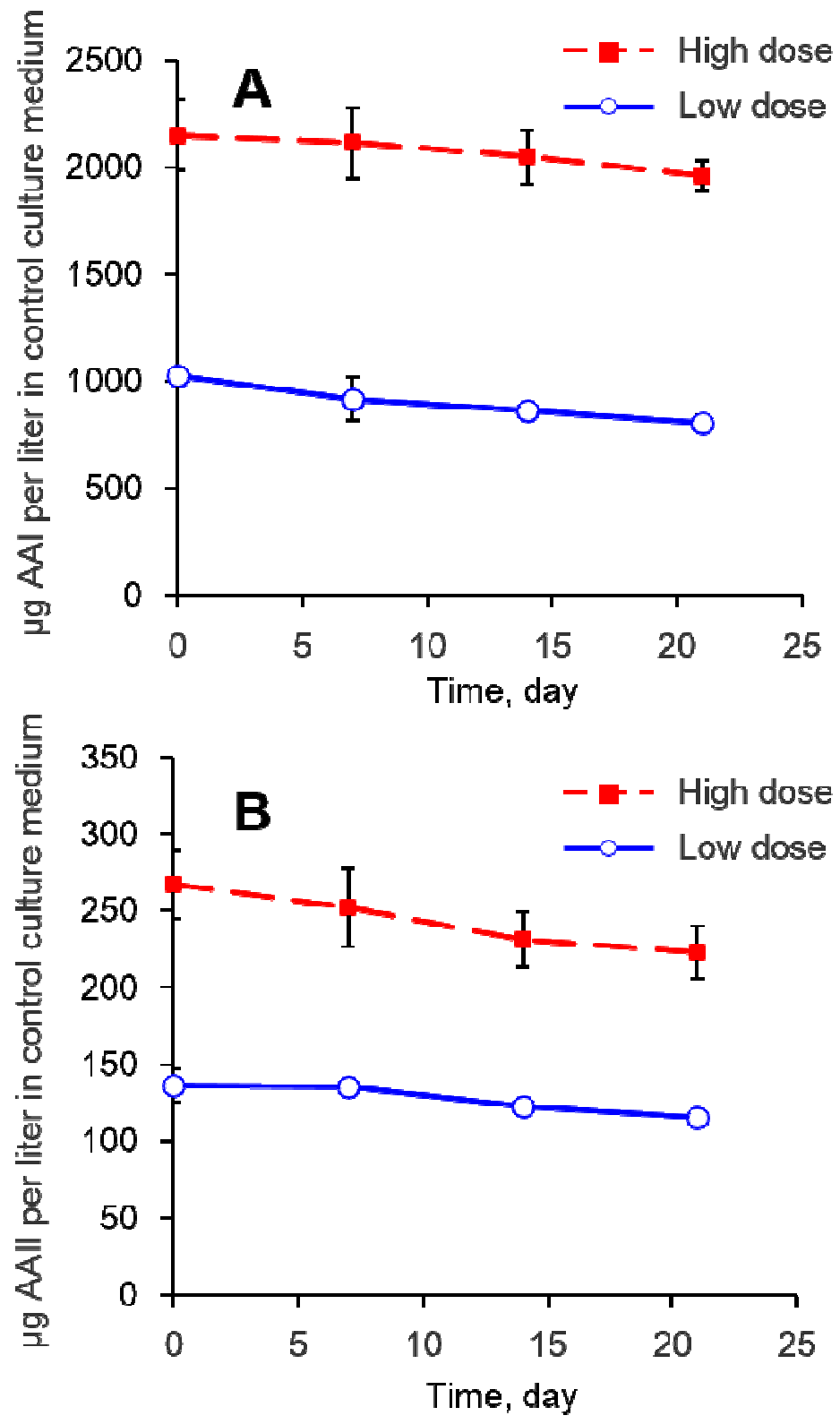